\title{
Klebsiella pneumoniae: resistance to carbapenems carbapenemase-mediated in the area of Piacenza
}

Massimo Confalonieri', Enrica Cavatorta', Camilla Reboli', Maria Milanda Bertelli', Rossana Chiarabini', Daniela Padrini', Francesca Riggio', Corrado Confalonieri², Enrica Poggi'

I Laboratorio Analisi Chimico-Cliniche e Microbiologia, Presidio Ospedaliero di Piacenza

2 Università degli Studi di Parma, Facoltà di Farmacia

Key words: Klebsiella pneumoniae, Carbapenemase, Phenotypic test.

Klebsiella pneumoniae: sorveglianza della resistenza ai carbapenemi mediata dalla produzione di carbapenemasi nella provincia di Piacenza

\section{SUMMARY}

The detection of Klebsiella pneumoniae isolates showing a reduced susceptibility or resistance to carbapenems represents an increasing problem.

The aim of this study is to survey the presence of carbapenemases producing strains in our country.

22 isolates of $K$. pneumoniae isolates showing carbapenems MIC => 2 by Vitek 2 System, were collected in the period May-August 2010 and studied for carbapenemases production using phenotypic confirmatory tests.

The modified Hodge test and the DD-sinergy test with boronic acid yelded positive results for 17/22 strains; all the isolates resulted negative to the E-test MBL (imipenem and imipenem/EDTA).

The results of this study confirm the recent emergence of KPC-producing K. pneumoniae strains in the area of Piacenza. The phenotypic tests employed appear reliable and simple for the confirmation of carbapenemases-production in $K$. pneumonie.

\section{INTRODUZIONE}

La resistenza degli enterobatteri agli antibiotici beta-lattamici è in costante aumento; ceppi produttori di beta-lattamasi a spettro esteso risultano ampiamente diffusi e le segnalazioni di enterobatteri resistenti ai carbapenemi, in particolare in Klebsiella pneumoniae sono in crescita.

La resistenza a questa classe di antibiotici può essere determinata dalla produzione di carbapenemasi di classe A (KPC) o di classe $\mathrm{B}(\mathrm{MBL})$.Una ridotta sensibilità ai carbapenemi può anche essere determinata dalla produzione di ESBL in associazione con meccanismi di impermeabilità della membrana batterica (deficit di porine).

Lo studio si prefigge, a seguito della segnalazione della presenza di ceppi produttori di carbapenemasi anche in Italia,di valutare la diffusione di isolati di $K$. pneumoniae resistenti ai carbapenemi nel nostro territorio. Tale resistenza potrebbe essere messa in relazione al crescente impiego di carbapenemi per la terapia delle infezioni da Gram negativi multiresistenti.

\section{MATERIALI E METODI}

Nel periodo Maggio-Agosto 2010 sono stati raccolti 22 ceppi di $K$. pneumoniae che all'analisi della sensibilità agli antibiotici effettuata mediante il sistema automatizzato in uso, mostravano resistenza o ridotta sensibilità ai carbapenemi (MIC $=>2 \mu \mathrm{g} / \mathrm{ml}$ ). Identificazioni ed antibiogrammi sono stati eseguiti utilizzando il sistema Vitek 2 (software AES 4.01), Card GN, AST-N089. Tutti gli isolati sono stati successivamente sottoposti al test di Hodge modificato per la valutazione presuntiva della produzione di carbapenemasi (2).

I ceppi risultati positivi al precedente test sono stati caratterizzati ulteriormente a livello fenotipico mediante i test di sinergia con acido boronico e con EDTA al fine di valutare, rispettivamente, se vi fosse produzione di enzimi di tipo KPC o di tipo MBL (1).

Il test di Hodge modificato (MHT) è stato eseguito sempre inoculando in $\mathrm{MH}$ agar una sospensione di $E$. coli ATCC 25922 pari a $0.5 \mathrm{McF}$ diluita $1: 10$ con fisiologica $0.85 \%$ e ponendo al centro della piastra un dischetto di meropenem $(10 \mu \mathrm{g})$. L'isolato da caratterizzare è stato quindi inoculato in linea retta utilizzando un ansa da $10 \mu \mathrm{l}$ a partire dal dischetto di meropenem e fino al margine della piastra. Dopo $18-24$ ore di incubazione a $35^{\circ} \mathrm{C}$ la presenza di una modifica- zione dell'alone di inibizione (smussatura di crescita) nel punto di intersezione fra alone di inibizione ed isolato saggiato è stato ritenuto indicativo di positività al test (Figura I).

Il test per valutare la produzione di MBL è stato eseguito inoculando in $\mathrm{MH}$ agar una sospensione pari a $0.5 \mathrm{McF}$ del ceppo in esame ed applicando una striscia di E-test MBL (bioMérieux). Dopo $18-24$ ore di incubazione a $35^{\circ} \mathrm{C}$, un rapporto $=>8$ delle MIC dei due reagenti presenti nella striscia, la formazione di una zona "fantasma" o la deformazione di una delle due ellissi di inibizione sono stati considerati indicativi della presenza di un ceppo produttore di metallo betalattamasi (Figura II).

Per valutare la produzione di carbapenemasi di tipo KPC è stato adottato il test di sinergia con acido boronico (PBA 250 $\mu \mathrm{g}$ - Rosco Diagnostica). Dopo l' inoculo in MH agar di un sospensione di $0.5 \mathrm{McF}$ del ceppo in esame, un dischetto di imipenem (10 $\mu \mathrm{g}$-Oxoid) ed uno di meropenem (10 $\mu \mathrm{g}$-Oxoid $)$ sono stati collocati ai due lati (6 $\mathrm{mm}$ da margine a margine) di un dischetto di PBA posizionato al centro della piastra. La presenza, dopo $18-24$ ore di incubazione a $35^{\circ} \mathrm{C}$, di un effetto sinergico a livello di uno o entrambi i dischetti dei carbapenemi è stato considerato indicativo della presenza di KPC (Figura III). Un ceppo di K. pneumoniae KPC produttore caratterizzato a livello molecolare presso l'Istituto di Microbiologia della Facoltà di Medicina e Chirurgia dell'Università di Pavia è stato utilizzato come controllo positivo.

\section{RISULTATI}

Nel periodo considerato sono stati isolati, da pazienti diversi, 22 ceppi di K. pneumoniae che mostravano resistenza o con ridotta sensibilità ai carbapenemi; di questi 7 provenivano da urine, 5 da sangue, 4 da materiali respiratori, 3 da tamponi cutanei, 2 da drenaggi, 1 da coltura di CVC.

Il test di Hodge modificato (MHT) è risultato positivo per 17/22 ceppi saggiati. Tutti i 17 ceppi sono risultati positivi al DDST per la rivelazione della produzione di KPC, mentre nessuno degli stessi isolati è risultato positivo al test per la rilevazione delle MBL (MBLT).

I valori di MIC per l'imipenem sembrano correlare bene con la positività al test $\mathrm{MHT}$ : nessuno dei tre isolati con $\mathrm{MIC}=2$ è risultato positivo a tale test, mentre $17 / 18$ isolati con MIC $=>8$ si sono confermati positivi al test $\mathrm{MHT}$ (Tabella 1).

\section{Corresponding author: Confalonieri Massimo}

Indirizzo: Laboratorio Analisi Chimico-Cliniche e Microbiologia, Presidio Ospedaliero,

Via Taverna, 49 - 29I2I Piacenza - Tel.: 0523302415 -Fax 0523302439

E-mail: m.confalonieri@ausl.pc.it 


\section{CONCLUSIONI}

Questo studio mette in evidenza la comparsa di ceppi di $K$. pneumoniae produttori di carbapenemasi di tipo KPC anche nella nostra area geografica. Dall'analisi dei fenotipi di resistenza degli isolati si può ipotizzare la circolazione intraospedaliera di un ceppo clonale di K. pneumoniae.

Tale ipotesi potrà essere confermata da caratterizzazioni genotipiche tuttora in corso.

Le modalità suggerite per la rilevazione presuntiva di ceppi produttori di carbapenemasi risultano di facile impiego e in grado di fornire preziose informazioni utili sia al fine di indirizzare correttamente il clinico nella scelta terapeutica, sia nell'ottica del contenimento della diffusione ospedaliera di batteri in grado di trasferire questo meccanismo di resistenza.

Tabella I.

\begin{tabular}{|c|c|c|c|c|c|c|c|c|c|}
\hline $\mathbf{N}^{\circ}$ & Materiale & Reparto & MIC Imipenem & MIC Ertapenem & MIC Meropenem & AES Vitek2 & MHT & MBLT & DDST \\
\hline $\mathrm{I}$ & Broncoasp & Rianimazione & $8(\mathrm{I}-\mathrm{R})$ & $>=8 R$ & $2(S-R)$ & $\mathrm{imp} / \mathrm{esbl+c}$ & + & - & + \\
\hline 2 & Urine & Chirurgia & $>=16 R$ & $>=8 \mathrm{R}$ & $8(\mathrm{I}-\mathrm{R})$ & imp/esbl+c & + & - & + \\
\hline 3 & Urine & Rianimazione & $>=16 R$ & $>=8 R$ & $8(\mathrm{I}-\mathrm{R})$ & imp/esbl+c & + & - & + \\
\hline 4 & Escreato & Rianimazione & $8(\mathrm{I}-\mathrm{R})$ & $>=8 R$ & $>=16 R$ & imp/esbl+c & + & - & + \\
\hline 5 & Drenaggio & Chirurgia & $>=16 R$ & $>=8 R$ & $>=16 R$ & imp/esbl+c & + & - & + \\
\hline 6 & Sangue & Rianimazione & $>=16 R$ & $>=8 R$ & $8(\mathrm{I}-\mathrm{R})$ & $\mathrm{imp} / \mathrm{esbl+c}$ & + & - & + \\
\hline 7 & Urine & Medicina CSG & $>=16 R$ & $>=8 \mathrm{R}$ & $8(\mathrm{I}-\mathrm{R})$ & imp/esbl+c & + & - & + \\
\hline 8 & Sangue & Rianimazione & $8(\mathrm{I}-\mathrm{R})$ & $>=8 R$ & $2(S-R)$ & imp/esbl+c & + & - & + \\
\hline 9 & Sangue & Chirurgia & $>=16 R$ & $>=8 R$ & $>=16 R$ & imp/esbl+c & + & - & + \\
\hline 10 & CVC & Rianimazione & $>=16 R$ & $>=8 \mathrm{R}$ & $>=16 R$ & imp/esbl+c & + & - & + \\
\hline II & Urine & Ginecologia & $2(S-R)$ & $>=8 R$ & $>=16 R$ & imp/esbl+c & - & & \\
\hline 12 & Urine & Rianimazione & $2(S-R)$ & $>=8 \mathrm{R}$ & $4(S-R)$ & $\mathrm{imp} / \mathrm{esbl+c}$ & - & & \\
\hline 13 & Urine & Esterno CSG & $>=16 R$ & $>=8 \mathrm{R}$ & $8(\mathrm{I}-\mathrm{R})$ & imp/esbl+c & + & - & + \\
\hline 14 & Urine & Gastroenterol & $>=16 R$ & $>=8 R$ & $>=16 R$ & imp/esbl+c & - & & \\
\hline 15 & Sangue & Geriatria & $>=16 R$ & $>=8 \mathrm{R}$ & $>=16 R$ & imp/esbl+c & + & - & + \\
\hline 16 & Drenaggio & Chirurgia & $>=16 R$ & $>=8 \mathrm{R}$ & $8(\mathrm{I}-\mathrm{R})$ & $\mathrm{imp} / \mathrm{esbl+c}$ & + & - & + \\
\hline 17 & Sangue & Rianimazione & $>=16 R$ & $>=8 \mathrm{R}$ & $>=16 R$ & imp/esbl+c & - & & \\
\hline 18 & Escreato & Rianimazione & $2 S(>16 R)$ & $>=8 \mathrm{R}$ & $>=16 R$ & Carbapenem & - & & \\
\hline 19 & Tamp cute & Rianimazione & $>=16 R$ & $>=8 \mathrm{R}$ & $>=16 R$ & imp/esbl+c & + & - & + \\
\hline 20 & Escreato & Rianimazione & $>=16 \mathrm{R}$ & $>=8 \mathrm{R}$ & $8(\mathrm{I}-\mathrm{R})$ & imp/esbl+c & + & - & + \\
\hline 21 & Ferita chir & Chirurgia & $>=16 R$ & $>=8 \mathrm{R}$ & $8(\mathrm{I}-\mathrm{R})$ & imp/esbl+c & + & - & + \\
\hline 22 & Tamp cute & Chirurgia & $>=16 R$ & $>=8 R$ & $8(\mathrm{I}-\mathrm{R})$ & imp/esbl+c & + & - & + \\
\hline
\end{tabular}

MHT:Test di Hodge modificato; MBLT: test per la determinazione di metallo-beta-lattamasi; DDST: double-DD synergy test per il rilievo di KPC; Imp: Resistente ai carbapenemi (Impermeabilità); esbl+c: ESBL+Carbapenemasi;

Tra parentesi sono riportate le modifiche suggerite dal sistema esperto (AES-Vitek2)

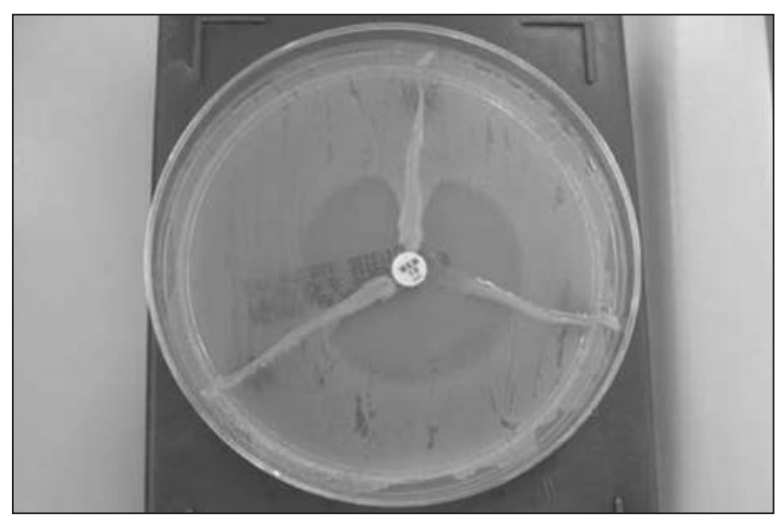

Figura I. Test di Hodge modificato.

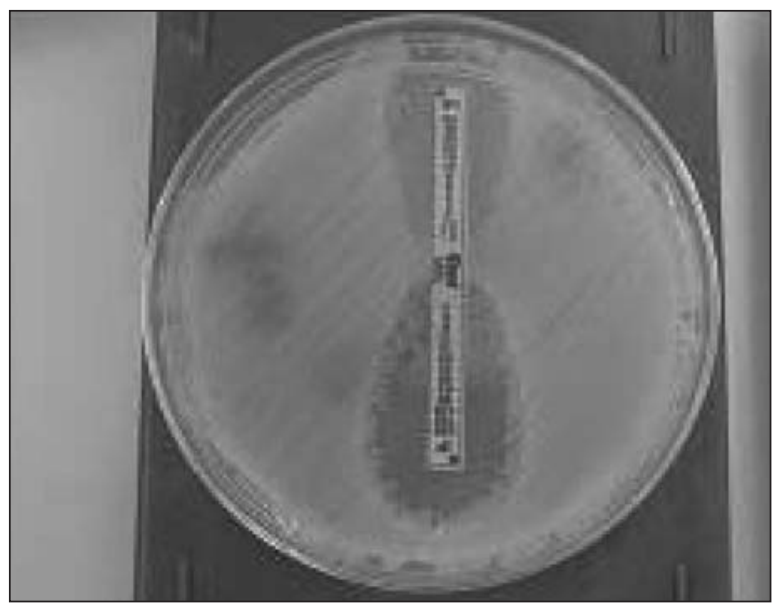

Figura II. Test fenotipico per la valutazione della produzione di MBL.

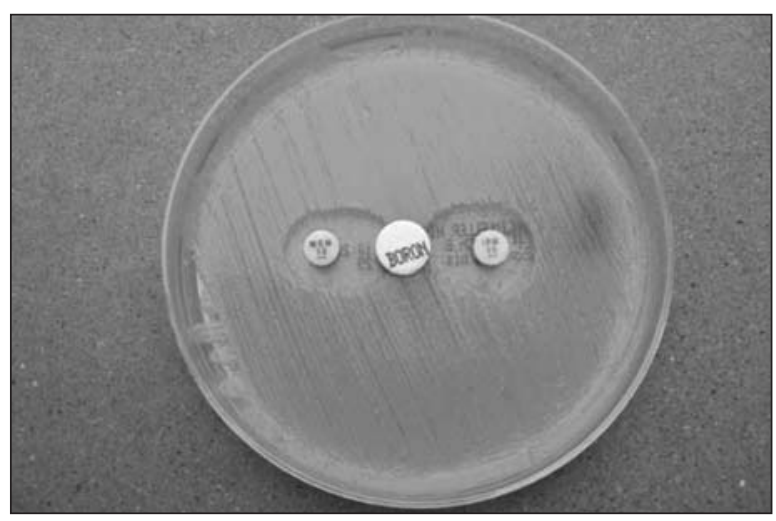

Figura III. Test fenotipico adatto allo screening della produzione di carbapenemasi di tipo KPC in K. pneumoniae.

\section{RINGRAZIAMENTI}

Si ringraziano il Direttore della sezione di Microbiologia della Facoltà di Medicina e Chirurgia dell'Università degli Studi di Pavia Prof.ssa L. Pagani e la Dott.ssa R. Migliavacca per la collaborazione prestata alla realizzazione di questo lavoro.

\section{BIBLIOGRAFIA}

1. Anderson K, Lonsway DR, Rasheed JK, Biddle J, et al. Evaluation of methods to identify the Klebsiella pneumoniae carbapenemase in Enterobacteriaceae. J Clin Microbiol 2007; 45: 2723.

2. Clinical and Laboratory Standards Institute. Performance standards for antimicrobial susceptibility testing; 19th informational supplement. CLSI documents M100-S19. Clinical and Laboratory Standards Institute, Wayne, Pa. 2009CLSI 2009 Document M100-S19.

4. Pasteran F, Mendez T, Guerriero L, Rapoport M, Corso A. Sensitive screening tests for suspected Class A Carbapenemase production in species of Enterobacteriaceae. J Clin Microbiol. 2009; 47: 1631-9. 